\title{
Effect of Multi-Frequency Beam Alignment on Non-Line-of-Site Vehicle to Infrastructure Communication using CI Model (CI-NLOS-V2I)
}

\author{
Mahmoud Zaki Iskandarani \\ Faculty of Engineering \\ Al-Ahliyya Amman University \\ Amman, Jordan
}

\begin{abstract}
Investigation of the effect of beam alignment for milimeter wave (mmWave) transmission in the case of Vehicleto-Infrastructure communication (V2I) is carried out. The investigation covered varying transmission-reception (TX-RX) distances. The effect of carrier frequency variation using different antenna angles and gains is also analyzed. The results showed convergence of path loss (PL) values regardless of angle or antenna gain $(\mathrm{dBi})$. The investigation also proved that shadow fading (SF), which is related to standard deviation $(\sigma)$ and exponent number (n) is a main contributor to the observed high path loss values in the case of misalignment. It is also noted that the path loss values decreases as a function of frequency per same travelled distance, which is related to the exponent number. This work highlights the importance of antenna alignment and that V2I communication can be very much optimized if and when auto-antenna alignment is used, and the importance of multiantenna arrays.
\end{abstract}

Keywords-Intelligent transportation systems; autonomous vehicles; connected vehicles; mmWave; channel model; path loss; CI Model; NLOS; V2I; V2V

\section{INTRODUCTION}

Connected and autonomous vehicles will play a pivotal role intelligent transportation systems in smart cities and will to a large extent depend on connectivity and communications between vehicles, hence high speed communicating nodes with low latency wireless links are critical for the success of applying the concept of smart cities. Connectivity among vehicles is of prime importance, as it contributes greatly to enhance roads safety, reduce environmental effects and provide higher level of living. Such objectives can only be achieved through provision of smart sensors with ability to transmit signals directly or through the cloud at high bit rates.

Mobile communications employing Millimeter wave (mmWave) is seen as a promising frequency band for the mobile, vehicular wireless networking as it is expected to have large spectrum. It is foreseen that it will have excellent potential in reaching extremely high data rates, which will exceed by far traditional cellular systems operating at sub-6 $\mathrm{GHz}$ bands [1-4].

Recently, a great interest in the design and development of vehicles capable of driving autonomously is realized and supported by research institutes and industrial organization.
Autonomous and connected vehicles and their driving technologies are important research topics in the automotive world. The vehicle technologies available at present are only a small sample of what to expect for the future. Technologies based on driver assistance are under development and directed towards autonomous vehicles (AS) and connected vehicles (CV) [5-6].

Connected vehicles are vehicles that employ various communication techniques to interface with the driver, other vehicles, roadside, and the Cloud. Theses interfaces can be used to improve vehicle safety, efficiency and commuting times. In general, vehicle communication comprises exchanging of information from a vehicle to any entity that may contribute to the functionality of the vehicle. They provide critical information to a driver or a vehicle in order to assist taking better actions. Connected vehicles principles of operation does not involve the vehicle making any decisions on behalf of the driver, contrary to Autonomous Vehicles.

Many autonomous vehicles currently in development are based on a sensory processing systems, communicating through an On Board Units (OBUs). The provision of sensors and wireless communication devices in an increasing number of vehicles is aimed at exchanging data with the vehicles, providing information to the vehicles and drivers, and collecting critical information about the vehicles activities.

\section{BACKGROUND}

The exponential increase of vehicles with OBUs could cause an overload on the cellular network, hence, a degradation of the quality of service (QoS). For vehicle-to-vehicle (V2V) and vehicle-to-infrastructure (V2I) communications, particular attention is given to the wireless access in vehicular environments [7-8].

Thus, the need for higher capacity in mobile communications is on the increase. The $5 \mathrm{G}$ wireless standard is trying to resolve bandwidth critical issues, such as bottleneck by including extra features like ubiquitous connectivity, significantly lower latency, and ultra-high-speed data transfers.

To achieve these objects, a more efficient use of the spectrum is required over the employed wireless communication frequency, thus leading to the concept of millimeter-wave (mmWave) [9-11]. 
In order to work with mmWave, a detailed knowledge of the communication channel and propagation path characteristics is essential, in order to provide the required reliable service. New challenges are faced working with very high frequencies, such as range, shadowing, fading and more importantly mobility and directional communication.

Recently, a marked progress is achieved in employing the mmWave in automotive applications, specifically in Vehicleto-Everything (V2X) channel propagation application and transmission modeling comprising Vehicle-to-Vehicle (V2V) and Vehicle-to-Infrastructure (V2I) communications. Such communication technology is crucial for intelligent transportation of the future that includes autonomous vehicles by transcending the current wireless communications limitations, thus enabling big data exchange for a V2X communication with internet of things (IoT) interface to the cloud.

The notable rise in demands for increased mobile data rates and distributed data access have led to a choice of alternative frequency bands with a fresh spectrum allocations using the millimeter wave (mmWave) frequency bands, which is a factor in supporting the $5^{\text {th }}$ generation (5G) wireless communication.

Non-Line-of-Sight (NLOS) work covering the range from above $6 \mathrm{GHz}$ and up to $100 \mathrm{GHz}$ is carried out over the past few years in relation to urban microcells (UMi) and urban macrocells (UMa) with directional and omnidirectional setups.

Intelligent Transportation Systems (ITS) will be able to connect vehicles and infrastructure at Gbps rate. ITS will depend on vehicle-to-everything (V2X) communications to share information among vehicles. However, mmWave communication suffers from beam alignment problems due to dynamic vehicle traffic, which causes regular beam misalignment which tends to degrade performance and contributes to routing problems.

In this paper, an investigation into the effect of beam alignment for V2I communication at various $\mathrm{GHz}$ frequencies is carried out. The frequency range used in this work covers 4 $\mathrm{GHz}$ to $100 \mathrm{GHz}$ range. Such spectrum is used in order to observe signal behavior change that covers all frequencies of interest in both V2I and V2V communication. A mathematical and parametric model is proposed to be used with the already established CI model [12-16].

\section{The CI PATHLOSS MODEL}

Different models developed that account for path loss namely;

1) Alpha-Beta-Gamma (ABG).

2) Close-in free space reference distance with frequency weighting (CIF).

3) Close-in (CI).

All developed models are appropriate in certain environments. Thus it is very important to understand that a selected model can be used in a new application whilst continue to possess stability, reliability, efficiency, and accuracy.
For V2I communication, it is concluded from previous studies that CI model is more fitted to describe such communication pattern due to its fewer variables that need to be controlled and the very good results it produces with high reliability. CI model $(\mathrm{dB})$, is given by the expression in equation (1).

$$
P L(f, d)=F S P L\left(f, d_{0}\right)+10 n \log _{10}\left(\frac{d}{d_{0}}\right)+\chi_{\sigma} .
$$

Where;

PL: Path loss, which is a function of Transmitter-Receiver separation distance (d)in meters between TX and RX over a frequency range in $\mathrm{GHz}$ and measured in $(\mathrm{dB})$

\section{f: Carrier frequency (GHz)}

FSPL: Free Space Path Loss (dB)

n: Path loss Exponent (PLE)

$\mathbf{d}_{\mathbf{0}}$ : Close-in free space reference distance (m)

$\chi_{\sigma}$ : Shadow fading which is zero-mean Gaussian random variable with a standard deviation $\sigma$ measured in $(\mathrm{dB})$

FSPL (dB) in equation (1) is given by the free space path loss in $\mathrm{dB}$ at a distance $\mathrm{d}_{0}$ given by Friis' free space in equation (2).

$$
\operatorname{FSPL}\left(f, d_{0}\right)=20 \log _{10}\left(\frac{4 \pi f d_{0} \times 10^{9}}{C}\right) .
$$

Where;

\section{c: Speed of light}

To show the contribution of the carrier frequency, equation (2) can be re-written as shown in equation (3).

$$
F S P L\left(f, d_{0}\right)=20 \log _{10}\left(\frac{4 \pi d_{0} \times 10^{9}}{c}\right)+20 \log _{10}(f)
$$
(4).

Equation (3), can be further simplified to produce equation

$$
\operatorname{FSPL}\left(f, d_{0}\right)=32.4+20 \log _{10}(f)
$$

Combining equations (1) and (4) yields equation (5):

$P L(f, d)=32.4+10 n \log _{10}\left(\frac{d}{d_{0}}\right)+20 \log _{10}(f)+\chi_{\sigma}$

The CI model provide a close-in free space reference for the path loss model to have reliable functionality of transmitted power in relation to covered distance. Also, consideration in the CI model is made to cover the main usage of the communication channels to be in the far field between $1 \mathrm{~m}$ and the Fraunhofer distance, due to the locations where the base station (BS) antennas will be mounted. (CI) reference distance model has better accuracy, stability as compared with other models [17-20]. 
The close-in free space reference distance (CI) path loss model has a solid basis, and is simultaneously applicable for frequencies both below and above $6 \mathrm{GHz}$ which is of interest to many applications as a step into the core of the mmWave environment. In addition, the CI path loss model demonstrates optimum parameter stability and forecasting accuracy for probable use over distances different to the original measurement range. It is favorable among other models to be used in urban microcell (UMi), urban macrocell (Uma), indoor hotspot ( $\mathrm{InH}$ ) among others compared to other path loss models with recent adoption in rural microcell (RMi) and macrocell (RMa) environments.

\section{Methodology}

The main objective of this work is to study the application of the CI model on mmWaves over a frequency range $4 \mathrm{GHz}$ $100 \mathrm{GHz}$ for V2I communication and the contribution of the following parameters through the use of data obtained at different angles: $\theta_{\text {new }}=\theta_{\text {old }}+\Delta \theta$ :

1) Effect of beam alignment between TX-RX.

2) Effect of Distance.

3) Effect of Carrier Frequency.

The approach followed in this work is based on firstly showing that when two different angled beams are aligned with their perspective transmitters through auto tracking, then their corresponding path loss values are the same or extremely close.

This can be achieved through re-writing equation (5) to obtain equation (6) [21-22].

$$
P L(f, d)=n \alpha+(\beta+32.4)+\chi_{\sigma}
$$

Where;

$$
\begin{aligned}
& \beta=20 \log _{10}(f) \ldots \\
& \alpha=10 \log _{10}\left(\frac{d}{d_{0}}\right)
\end{aligned}
$$

When beam alignment for two different transmitters and receivers at different angles is achieved, then all terms for both transmitters and receivers in equation (6) should equate.

The terms in equation (6) cover all three objectives mentioned as follows:

1. Effect of Alignment and Shadow fading (SF) $: \chi_{\sigma}$

2. Effect of distance: $\alpha=10 \log _{10}\left(\frac{d}{d_{0}}\right)$

\section{Effect of Frequency: $\beta=20 \log _{10}(f)$}

This will be proved through the obtained results and during discussion of the data obtained.

\section{RESULTS}

Table I presents the two components forming path loss (PL) due to free space (FSPL) over a frequency range $4 \mathrm{GHz}$ to $100 \mathrm{GHz}$, while Fig. 1 shows its characteristics.

Table II presents path loss values for two beam aligned situations at two different angles. The table presents two

\begin{tabular}{|c|c|c|}
\hline Frequency (f) GHz & FSPL (dB) & Frequency Contribution (dB) \\
\hline 4 & 44.48 & 12.08 \\
\hline 8 & 50.50 & 18.10 \\
\hline 12 & 54.03 & 21.63 \\
\hline 16 & 56.52 & 24.12 \\
\hline 20 & 58.46 & 26.06 \\
\hline 24 & 60.05 & 27.65 \\
\hline 28 & 61.38 & 28.98 \\
\hline 32 & 62.54 & 30.14 \\
\hline 36 & 63.57 & 31.17 \\
\hline 40 & 64.48 & 32.08 \\
\hline 44 & 65.31 & 32.91 \\
\hline 48 & 66.07 & 33.67 \\
\hline 52 & 66.76 & 34.36 \\
\hline 56 & 67.41 & 35.01 \\
\hline 60 & 68.00 & 35.60 \\
\hline 64 & 68.57 & 36.17 \\
\hline 68 & 69.09 & 36.69 \\
\hline 72 & 69.59 & 37.19 \\
\hline 76 & 70.06 & 37.66 \\
\hline 80 & 70.50 & 38.10 \\
\hline 84 & 70.93 & 38.53 \\
\hline 88 & 71.33 & 38.93 \\
\hline 92 & 71.72 & 39.32 \\
\hline 96 & 72.09 & 39.69 \\
\hline 100 & 72.44 & 40.04 \\
\hline
\end{tabular}
selected cases with the rest follow similar pattern.

TABLE I. Path Loss due to FreE Space

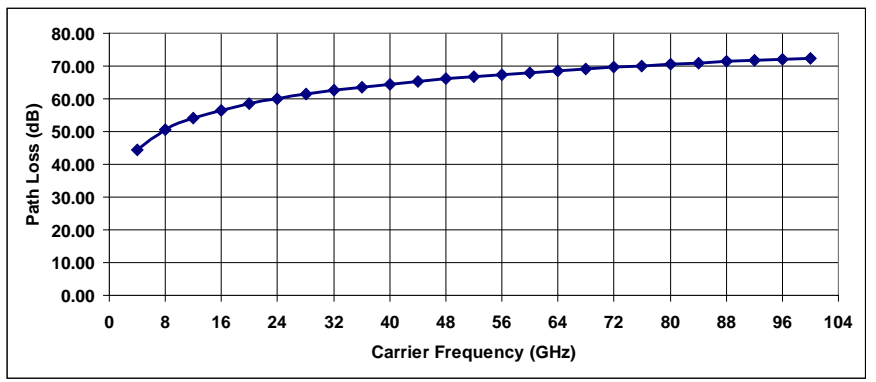

Fig. 1. Free Space Path Loss. 
TABLE II. PAth Loss Results BEAm Alignment TX-RX

\begin{tabular}{|c|c|c|c|c|}
\hline \multirow{2}{*}{$\frac{\text { Freq. }}{\text { GHz }}$} & \multicolumn{2}{|c|}{ Path Loss (dB) $\theta 1$} & \multicolumn{2}{|c|}{ Path Loss (dB) $\theta 2$} \\
\hline & $200 \mathrm{~m}$ & $300 \mathrm{~m}$ & $200 \mathrm{~m}$ & $300 \mathrm{~m}$ \\
\hline 4 & 126.9 & 133.2 & 126.8 & 133.0 \\
\hline 8 & 126.1 & 131.9 & 126.0 & 131.7 \\
\hline 12 & 125.7 & 131.1 & 125.5 & 130.9 \\
\hline 16 & 125.3 & 130.6 & 125.2 & 130.4 \\
\hline 20 & 125.1 & 130.2 & 124.9 & 130.0 \\
\hline 24 & 124.9 & 129.8 & 124.7 & 129.6 \\
\hline 28 & 124.7 & 129.5 & 124.5 & 129.3 \\
\hline 32 & 124.5 & 129.3 & 124.4 & 129.1 \\
\hline 36 & 124.4 & 129.0 & 124.2 & 128.9 \\
\hline 40 & 124.3 & 128.8 & 124.1 & 128.7 \\
\hline 44 & 124.2 & 128.7 & 124.0 & 128.5 \\
\hline 48 & 124.1 & 128.5 & 123.9 & 128.3 \\
\hline 52 & 124.0 & 128.3 & 123.8 & 128.2 \\
\hline 56 & 123.9 & 128.2 & 123.7 & 128.0 \\
\hline 60 & 123.8 & 128.1 & 123.7 & 127.9 \\
\hline 64 & 123.8 & 127.9 & 123.6 & 127.8 \\
\hline 68 & 123.7 & 127.8 & 123.5 & 127.6 \\
\hline 72 & 123.6 & 127.7 & 123.4 & 127.5 \\
\hline 76 & 123.6 & 127.6 & 123.4 & 127.4 \\
\hline 80 & 123.5 & 127.5 & 123.3 & 127.3 \\
\hline 84 & 123.4 & 127.4 & 123.3 & 127.2 \\
\hline 88 & 123.4 & 127.3 & 123.2 & 127.2 \\
\hline 92 & 123.3 & 127.3 & 123.2 & 127.1 \\
\hline 96 & 123.3 & 127.2 & 123.1 & 127.0 \\
\hline 100 & 123.2 & 127.1 & 123.1 & 127.0 \\
\hline
\end{tabular}

\section{Discussion AND CONCLUSIONS}

From Table $\mathrm{I}$ it is clear the contribution of the carrier frequency towards path loss as FSPL increases as a function of frequency, which is expressed by equation (6.1). Fig. 1 shows a plot of FSPL as a function of frequency.

By analyzing the contents of Table II and Fig. 2 and 3, the following is observed:

1) When the beams are aligned, path loss values are lower than when they are not aligned.

2) Path loss values increase with distance and affected mainly by equation (6.2).

3) Path loss values decreases as the carrier frequency increases. This is due mainly to the exponent (n) affecting the shadow fading (SF).

4) Path Loss values for different beams with aligned TX$\mathrm{RX}$ converge to almost same values. This can be explained by referring to equation (6).

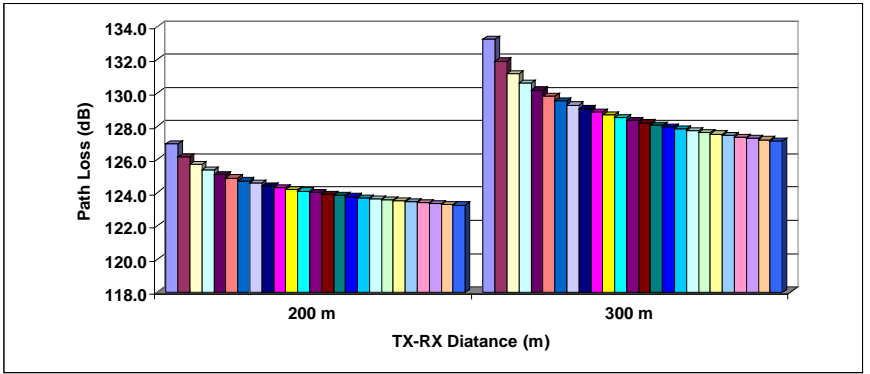

Fig. 2. Path Loss as a Function of Distance and Frequency for Beam aligned $\theta 1$.

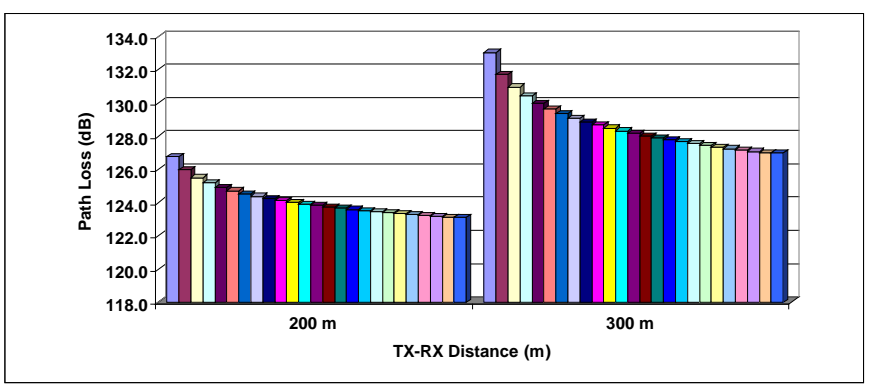

Fig. 3. Path Loss as a Function of Distance and Frequency for Beam aligned $\theta 2$.

Thus, all terms in equation (6) should equate as presented in equation. (7). Thus, fulfilling the conditions in a, b, and c below.

$P L(f, d)=n \alpha\left(\theta_{i}\right)+\left(\beta\left(\theta_{i}\right)+32.4\right)+\chi_{\sigma}$

Equal Distance:

$\beta\left(\theta_{1}\right)=\beta\left(\theta_{2}\right)$

Equal Frequency:

$\alpha\left(\theta_{1}\right)=\alpha\left(\theta_{2}\right)$

Free Space Path Loss is constant

So, the shadow fading $\left(\chi_{\sigma}\right)$ and the exponent (n) are to equate to support the obtained values in Table II and Fig. 2 and 3.

This indicates that when the beams are not aligned, the two factors that could contribute to higher and different path loss values for equal frequency, distance, and FSPL, are $\left(\chi_{\sigma}\right)$ and exponent (n).

These findings point towards a change in the standard deviation of the shadow fading, which can be expressed by firstly rearranging equation (7) to obtain equation (8).

$P L(f, d)-\left(\beta\left(\theta_{i}\right)+32.4\right)-n \alpha\left(\theta_{i}\right)=\chi_{\sigma}$

Assume that:

$P L(f, d)-\left(\beta\left(\theta_{i}\right)+32.4\right)=\zeta\left(\theta_{i}\right)$

Then equation (8) becomes:

$\zeta-n \alpha\left(\theta_{i}\right)=\chi_{\sigma}\left(\theta_{i}\right)$ 
Now the standard deviation can be defined as:

$$
\sigma\left(\theta_{i}\right)=\sqrt{\frac{\left(\zeta-n \alpha\left(\theta_{i}\right)\right)^{2}}{M}}=\sqrt{\frac{\left(\chi_{\sigma}\right)^{2}}{M}}
$$

Where;

\section{M: Number of data points obtained during communication.}

Table III shows an infinitesimal but constant difference between the presented path loss values corresponding to two differently aligned beams with distance dependence and frequency independence.

From these values, the following is deduced:

1) The presented difference values can be approximated to zero as their $\mathrm{dB}$ values are within the approximation and margin errors.

2) Since such values have constant difference irrespective of carrier frequency, it shows that both aligned beams actually converged to almost equal path loss values.

TABLE III. PATH LOSS DIFFERENCE FOR $\theta 1: \theta$ I DEGRESS- BEAM ALIGNMENT

\begin{tabular}{|c|c|c|}
\hline Frequency & \multicolumn{2}{|c|}{ TX-RX Distance } \\
\hline $\mathrm{GHz}$ & $200 \mathrm{~m}$ & $300 \mathrm{~m}$ \\
\hline 4 & 0.17 & 0.19 \\
\hline 8 & 0.17 & 0.19 \\
\hline 12 & 0.17 & 0.19 \\
\hline 16 & 0.17 & 0.19 \\
\hline 20 & 0.17 & 0.19 \\
\hline 24 & 0.17 & 0.19 \\
\hline 28 & 0.17 & 0.19 \\
\hline 32 & 0.17 & 0.19 \\
\hline 36 & 0.17 & 0.19 \\
\hline 40 & 0.17 & 0.19 \\
\hline 44 & 0.17 & 0.19 \\
\hline 48 & 0.17 & 0.19 \\
\hline 52 & 0.17 & 0.19 \\
\hline 56 & 0.17 & 0.19 \\
\hline 60 & 0.17 & 0.19 \\
\hline 64 & 0.17 & 0.19 \\
\hline 68 & 0.17 & 0.19 \\
\hline 72 & 0.17 & 0.19 \\
\hline 76 & 0.17 & 0.19 \\
\hline 80 & 0.17 & 0.19 \\
\hline 84 & 0.17 & 0.19 \\
\hline 88 & 0.17 & 0.19 \\
\hline 92 & 0.17 & 0.19 \\
\hline 96 & 0.17 & 0.19 \\
\hline 100 & 0.13 & 0.11 \\
\hline
\end{tabular}

Thus it can be stated with certainty that, when different beams are aligned, they will suffer almost equal path loss under similar conditions, regardless of angle or antenna gain $(\mathrm{dBi})$.

So from equation (9):

$$
\frac{d \chi_{\sigma}\left(\theta_{1}\right)}{d \chi_{\sigma}\left(\theta_{2}\right)}=0
$$

From equation (10):

$$
\frac{d \sigma\left(\theta_{1}\right)}{d \sigma\left(\theta_{2}\right)}=0
$$

Where;

$\theta_{1}, \theta_{2}$ : Angles of two different antennas having beam alignment.

\section{REFERENCES}

[1] T. Hong, J. Yao, C. Liu, F. Qi "mmWave Measurement of RF Reflectors for 5G Green Communications,” Wireless Communications and Mobile Computing, vol. 2018, Article ID 8217839, pp. 1-10, 2018.

[2] A. Hamed, R. Rao, "Spectral and Energy Efficiencies in mmWave Cellular Networks for Optimal Utilization,” Wireless Communications and Mobile Computing, vol. 2018, Article ID 3097094, pp. 1-11, 2018.

[3] R. Magueta, V. Mendes, D. Castanheira, A. Silva, R. Dinis, A. Gameiro1, "Iterative Multiuser Equalization for Subconnected Hybrid mmWave Massive MIMO Architecture, vol. 2017, Article ID 9171068, pp. 1-13, 2017.

[4] R. Santos, K. Koslowski, J. Daube, H. Ghazzai, A. Kassler, K. Sakaguchi, T. Haustein, "mmWave Backhaul Testbed Configurability Using Software-Defined Networking," Wireless Communications and Mobile Computing, vol. 2019, Article ID 8342167, pp. 1-24, 2019.

[5] X. Fan, T. Cui, C. Cao, Q. Chen, K. Kwak, "Minimum-Cost Offloading for Collaborative Task Execution of MEC-Assisted Platooning” Sensors, vol. 12, pp. 1-16, 2019.

[6] Y. Zhou, S. Liu, M. Xiao, S. Deng, X. Wang, “An Efficient V2I Authentication Scheme for VANETs," Mobile Information Systems, vol. 2018 Article ID 4070283, pp. 1-11, 2018.

[7] C. Campolo, R. dos Reis Fontes, A. Molinaro, C. Rothenberg, A. Iera, "Slicing on the Road: Enabling the Automotive Vertical through 5G Network Softwarization” Sensors, vol. 18, no. 4435, pp. 1-17, 2018.

[8] P. Sewalkar, J. Seitz, "Vehicle-to-Pedestrian Communication for Vulnerable Road Users: Survey, Design Considerations, and Challenges,” Sensors, vol. 19, no. 358, pp. 1-18, 2019.

[9] S.Busari, M. Khan, K. Saidul Huq, S. Mumtaz, J. Rodriguez "Millimetre-wave Massive MIMO for Cellular Vehicle-to-Infrastructure (C-V2I) Communication,” IET Intelligent Transport Systems, vol. 13, no. 6 pp. 983-990, 2019.

[10] S. Mignardi, C. Buratti, A. Bazzi, R. Verdone "Trajectories and Resource Management of Flying Base Stations for C-V2X,” Sensors, vol. 19, no. 811, pp. 1-15, 2019.

[11] M. Baek, D. Jeong, D. Choi, S. Lee, "Vehicle Trajectory Prediction and CollisionWarning via Fusion of Multisensors and Wireless Vehicular Communications,” Sensors, Vol. 20, No. 288, pp. 1-26, 2020.

[12] M. Attiah , A. Isa , Z. Zakaria ,M. Abdulhameed, M. Mohsen , A. Dinar "Independence and Fairness Analysis of 5G mmWave Operators Utilizing Spectrum Sharing Approach,” Mobile Information Systems, vol. 2019, Article ID 4370847, pp. 1-12, 2019.

[13] F. Qamar, N. Hindia, K. Dimyati, K. Noordin, M. Majed, T. Abd Rahman , I. Amiri, “Investigation of Future 5G-IoT Millimeter-Wave Network Performance at $38 \mathrm{GHz}$ for Urban Microcell Outdoor Environment,” Electronics, vol. 7, no. 495, pp. 1-26, 2019.

[14] Srinivas, B. Kumari, "Implementation of an Efficient Channel Model for 5G mm-Wave Bands," International Journal of Innovative 
Technology and Exploring Engineering, vol. 8, no. 12, pp. 1674-1678, 2019.

[15] A. Al-Samman, M. Azmi, Y. Al-Gumaei, T. Al-Hadhrami, T. Abd. Rahman, Y. Fazea, A. Al-Mqdashi, "MillimeterWave Propagation Measurements and Characteristics for 5G System," Applied Sciences, vol. 10, no.335, pp. 1-17, 2020.

[16] G. Gougeon, Y. Corre, M. Aslam, "SUB-THz Channel Characterization from Ray-Based Determinstic Simulations," ITU Journal: ICT Discoveries, vol. 2, no. 1, pp. 1-9, 2019.

[17] S. Sun, T. Rappaport, T. Thomas, A. Ghosh, H. Nguyen, I. Kov'acs, I. Rodriguez, O. Koymen, A. Partyka, "Investigation of Prediction Accuracy, Sensitivity, and Parameter Stability of Large-Scale Propagation Path Loss Models for 5G Wireless Communications," Journal of Advanced Transportation, IEEE Transactions on Vehicular Technology, vol. 65, no. 5, pp. 1-18, 2016.

[18] Y. Zhang, C. Anderson, N. Michelusi, D. Love, K. Baker, J. Krogmeier, "Propagation Modeling Through Foliage in a Coniferous Forest at 28 GHz,” IEEE Wireless Communications Letters, vol. 8, no. 3, pp. 901904, 2019.
[19] T. Rappaport, Y. Xing, G. MacCartney, A. Molisch, E. Mellios, J. Zhang, "Overview of Millimeter Wave Communications for FifthGeneration (5G) Wireless Networks-With a Focus on Propagation Models,” IEEE Transaction on Antennas and Propagation, vol65, no. 12, pp. 6213-6230, 2017.

[20] J. Lee, J. Liang, M. Kim, J. Park, B. Park, H. Chung "MeasurementBased Propagation Channel Characteristics for Millimeter-Wave 5G Giga Communication Systems,” ETRI Journal, vol. 38, no. 6 pp. 10311041, 2016.

[21] N. Srinivas, B. Kumari "Implementation of an Efficient Channel Model for 5G mm-Wave Bands, " International Journal of Innovative Technology and Exploring Engineering, vol. 8, no. 12, pp. 1674-1678, 2019.

[22] B. Antonescu, M. Tehrani, S. Basagni, "mmWave Channel Propagation Modeling for V2X Communication Systems", IEEE 28th Annual International Symposium on Personal, Indoor, and Mobile Radio Communications (PIMRC), 978-1-5386-3531-5/17, 2017 IEEE. 